\author{
Sharon O'Brien*, Maureen Ehrensberger-Dow**, Marcel Hasler** \& \\ Megan Connolly*
}

\title{
Irritating CAT Tool Features that Matter to Translators
}

\begin{abstract}
CAT tools have become a fixture of professional translation over the last two decades yet are still treated with suspicion or disinterest by many freelancers. Acknowledged to contribute to consistency and speed, they can constrain and otherwise negatively affect the translation process in various ways. Surveys of professional translators and observations at the workplace suggest that there is a degree of frustration associated with the use of CAT tools and room for improvement in their usability. A recent large-scale survey of professional translators included specific items for CAT tool users about whether any features of their tools were irritating or missing. Many reported that there were and also availed themselves of the opportunity to provide detailed comments about them. More than half of the CAT tool users said that they found some features irritating, and a quantitative and qualitative analysis of their comments revealed that the most common issues concerned the complexity of the user interface and segmentation. There were some differences in the responses between freelance, institutional and commercial translators but almost none across age groups. The comments about missing features also tended to be about making the tools easier to use. The focus in the survey reported here was on identifying negative aspects of tools with a view to mitigating them and tailoring the tools more to translators' needs. However, there is also room for research exploring the positive aspects of tools in the interests of optimising their usability and reducing cognitive friction.
\end{abstract}

\section{Keywords}

CAT tools, cognitive friction, irritating features, missing features, survey, professional translators, comments, usability

\section{Introduction}

After nearly two decades of development and deployment of Computer-Aided-Translation (CAT) tools, it could be expected that those tools are so entrenched in some translation workflows, and so well-accepted, that they would not warrant yet another paper on the topic. However, research in the past decade has indicated that translators, the users of CAT tools, are not entirely satisfied with the CAT tools they choose to use or are obliged to use. In Section 3 of this paper, a brief overview of related research demonstrates that while CAT tools have undoubtedly delivered significant benefits to translators, they are still a source of irritation to their users. In previous research (Ehrensberger-Dow/O'Brien 2015), we have conceptualised this irritation as 'cognitive friction', borrowing the term from Cooper (2004). Cognitive friction can be caused by organisational, task, or technological issues and occurs when a state of 'flow' is disturbed. We elaborate on this concept further in Section 2 of this paper. Our assumption was that CAT tools can be a source of cognitive friction, or irritation, and we sought to investigate this assumption by surveying professional translators, as explained in Section 4.

The primary research questions in the present study were:

* Sharon O'Brien \& Megan Connolly School of Applied Language \& Intercultural Studies

Dublin City University

sharon.obrien@dcu.ie
** Maureen Ehrensberger-Dow \& Marcel Hasler Zurich University of Applied Sciences ehre@zhaw.ch 
1. Which aspects or features of CAT tools are the most irritating for translators who use those tools?

2. What influence does the working environment have on the perception of these negative aspects?

3. What additional features do professional translators wish to have implemented in their CAT tools?

The data for the present study were obtained through an international online survey carried out as part of the ErgoTrans project, which focused on disturbances to the translation process and ergonomic factors that might be associated with them. ${ }^{1}$ That multi-disciplinary project used a variety of methods to explore the physical, cognitive and organisational ergonomic issues associated with the activity of professional translation. On-site ergonomic assessments, observations, videos and screen recordings were made at the workplaces of freelancers as well as those of translators working for institutions and commercial enterprises. The findings from the workplace phase of the project led to the refinement of an exploratory survey (see Ehrensberger-Dow/O'Brien 2015), especially with respect to the use of CAT tools and opinions about those tools. In Section 5 we present the findings from the quantitative and qualitative analysis of those survey data, the latter of which was carried out by the third and fourth authors under the supervision of the first two authors. Finally, we outline the conclusions and possible implications of this research in Section 6.

\section{Theoretical Underpinnings}

Cognitive friction was defined by Cooper (2004: 19) as "the resistance encountered by a human intellect when it engages with a complex system of rules that change as the problem changes." Although this definition is rather vague, we suggest that it can be useful for understanding irritation among translators in the highly technologized workplace (see also Ehrensberger-Dow/O'Brien 2015).

The concept of cognitive friction is linked to the concept of 'flow', which is used in psychology to refer to a state of being fully immersed in a task such that this immersion is energising (Nakamura/Csikszentmihalyi 2002). If the human encounters some resistance, or friction, in his or her task, then flow is interrupted. If this interruption in flow is caused by the tools the translator is using, then it manifests as general irritation with those tools. The survey reported here sought to investigate the extent to which translators reported irritation with the CAT tools they used.

Both of these concepts, cognitive friction and flow, are intrinsically linked to cognitive load. In a state of flow, the cognitive load is manageable for the human, and can even lead to enjoyment of the task. When something occurs to cause friction, for example, the CAT tool crashes, we speculate that cognitive load is impacted because the translator's flow is interrupted and a new problem has to be solved (restart the CAT tool, recover the position in the text, recall the translation solution, re-do some of the translation if not saved etc.).

Cognitive load theory has been mostly influential in the field of instructional psychology, where the focus is on what goes on in the mind of the learner when different input modalities are presented with a view to adapting instruction to a learner's cognitive system. The main motivation behind this line of inquiry in instructional psychology is that traditional instructional approaches do not take adequate account of a human's limited cognitive architecture and that, as a result, the learner's working memory can be overloaded (Schnotz/Kürschner 2007). Cognitive load is also a concept that has been used in translation process research, in particular in relation to post-editing of machine translation (e.g. O'Brien 2006, 2008, Carl et al. 2015). According to Schnotz/Kürschner (2007), neither the concept of cognitive load nor how it can be measured is still sufficiently clear.

1 More information about ErgoTrans can be found in Ehrensberger-Dow/Hunziker Heeb (2016) as well as on the project website (http://www.zhaw.ch/linguistics/ergotrans). 
Nonetheless, the identification of at least two types of cognitive load, intrinsic and extraneous load, could help us to understand the nature of the cognitive friction we investigate here. Intrinsic load is caused by the intrinsic nature of the task itself (in our case translation), while extraneous load is attributed to task-external aspects, such as operating system notifications, tool crashes or bugs. Extraneous load can also result from redundancy of information. In the case of a CAT tool, this could be incorrect or duplicated meta-data or too many suggestions being presented on the screen, or by those suggestions being unusable. As all load is assumed to be additive (Schnotz/ Kürschner 2007), we posit that cognitive friction arises when extraneous load due to unhelpful or distracting CAT tool features are added to the intrinsic load of the translation task itself.

Our understanding of cognitive friction arising from translators' interactions with non-optimal CAT tool features is consistent with an appreciation of translation as a situated activity and cognition as embedded, embodied, extended and/or distributed (Robbins/Aydede 2009). Risku (2002) was one of the first to discuss the situatedness of translation not only in terms of linguistic and cultural norms but also with reference to artefacts and tools involved in the production of target texts. More recently, Krüger (2016) has conceptualised CAT tools as forming part of what he terms the translational ecosystem and has called for more research into the usability of the tools. The empirical research reviewed in the next section identifies some of the problems that have limited the uptake of CAT tools and highlights the need to investigate their impact on the situated activity of professional translation.

\section{Related Research}

An example of an early study on translators' use of technology is Fulford/Granell-Zafra (2005) where it is reported that UK freelance translators seemed sceptical about the value of "translation-specific" software. Lagoudaki's survey of translators' use of Translation Memory (TM) systems $(2006,2008)$ was one of the first relatively large-scale international surveys on the topic of CAT tools. One of the conclusions from that survey was that "systems' usability and end-users' demands seem to have been of only subordinate interest" when translation memory systems were being designed and developed (Lagoudaki 2008: 17). In 2011, Olohan echoed this by pointing out that when translation tools were developed in the past, the human and organisational requirements were addressed only in an ad hoc manner at best.

In an ethnographic approach, LeBlanc (2013) investigated the impact of TM tools on translators in the workplace, with a focus on three language service providers in Canada. The translators who were interviewed mentioned both advantages and disadvantages of working with TM tools. Some of the advantages included increased productivity and consistency, as well as the elimination of repetitive work. Some disadvantages listed were the changed relationship with the text through forced segmentation, barriers to creativity and error propagation. LeBlanc noted that some of the dissatisfaction among translators "revolves around the tool's conception or design" (2013: 10), but he focused on segmentation as the primary issue.

In a similar ethnographic approach, eighteen staff translators working for a Swiss language service provider were observed intermittently at their workplaces over a period of about six months and their screen activity was recorded while they were doing non-confidential translations (Ehrensberger-Dow 2014, Ehrensberger-Dow/Massey 2014). An analysis of the screen recordings revealed that certain software features seemed to slow down the translation process and that the translators had to switch frequently between windows. In addition, some of the comments from the retrospective verbalisations of selected recordings related to the usability or ergonomics of the language technology tools they were using (Ehrensberger-Dow/Massey 2014: 76-78).

Such observations and comments led us (Ehrensberger-Dow/O'Brien 2015) to focus specifically on the ergonomics of the translation workplace, comparing survey responses from a small group of office-based translators in Switzerland to those of freelance translators in Ireland. The focus was purposely broad in that article, covering workplace conditions (i.e. physical ergonom- 
ics), sociotechnical aspects (i.e. organisational ergonomics), and health as well as issues associated with the use of technology (i.e. cognitive ergonomics).

Zaretskaya (2015) conducted a larger survey on working practices of professional translators and their level of satisfaction with technologies. The survey had 736 completed responses. When asked about which features were 'essential' or 'useful' versus 'not so useful', a significant number of the respondents labelled "high working speed" and "simple and intuitive interface, easy to learn" as "essential" features, which is relevant for our own results, presented below.

More recently, Moorkens/O'Brien (2016) carried out research with a view to developing specifications for editing tools that better supported the task of machine translation post-editing. A survey of professional translators was carried out, which was followed up by interviews with a smaller group of the respondents. In this survey, $75 \%$ of respondents reported using TM technology because it helps with their work, but only 56\% reported liking using it. Approximately half of the respondents reported being unhappy with the default layout of their TM tool and many also complained about performance issues (bugs, errors, slow response times). Although the research focused on UI design for post-editing, it was interesting to note that many of the responses highlighted a fundamental dissatisfaction with the basic TM technology being used.

In a survey study on the uptake of CAT tools in Denmark, Christensen/Schjoldager (2016) commented that CAT tools are seen to have had an impact on the status of translation, a worrying development since translator status was not high to begin with (Dam/Zethsen 2010). In open questions on the perceived impact of CAT tools, there were both positive and negative responses, e.g. increased productivity and quality vs. pressure on price, lower creativity.

Bundgaard et al. (2016) observed a translator at work with what they termed "MT-assisted TM" and concluded that the translator is both assisted by the tool and demonstrates resistance to the tool and how it forces a specific translation process on its user. In particular, the translator sometimes broke with the sequential segment-by-segment method imposed by the tool to attend to text-level cohesion.

This short review of related work demonstrates that there is an increasing interest in obtaining information from professional translators about their work conditions in general, but also specifically about their opinions on and interaction with CAT technology. The past decade has seen an increase in scholarly publications on the topic, many of which highlight issues and dissatisfaction with CAT tools, but some of which also highlight that translators see advantages to these tools. It is probably the case that many translators would lament not having a CAT tool to work with. At the same time, our impression is that translators are irritated by their tools and, given that the latter have been in use for over twenty years, it is time to address this frustration.

\section{Methodological Approach}

The approach used in this study is primarily qualitative in that verbal data in the form of comments provided in an online survey were analysed, though data are also presented from a quantitative perspective when appropriate. As sketched out above, there are various methodological approaches to investigating translators' interaction with CAT tools, including survey, ethnography, screen recording and eye tracking. The authors, among other researchers, have engaged with the latter two methods significantly (e.g., O’Brien 2006, 2008, Ehrensberger-Dow 2014), but focus here on the survey approach. The uniqueness of this online survey is that it was designed and pre-tested by an inter-disciplinary team that included practitioners, occupational therapists, and usability specialists in addition to translation studies researchers. The decision to have it available in several languages probably increased its reach and might have contributed to the large proportion of respondents who chose to provide additional information in the form of optional comments (see below). 
The analysis of the quantitative survey data related to the questions about CAT tools provides important context for interpreting the qualitative data. This is outlined in the next section, before the procedure for coding and categorising the comments is explained.

\subsection{Overview of the ErgoTrans survey}

The ErgoTrans survey was designed in collaboration with occupational therapy researchers to address potential issues at the translation workplace with respect to the various aspects of ergonomics as defined by the International Ergonomics Association (IEA 2016). There were a number of different sections meant to elicit information about the respondents' general background, workspace and environment, computer workstation, tools and resources, workflow and organization as well as health and related issues. The online survey was made available in six languages (English, German, Spanish, French, Italian and Portuguese), in order to increase the likelihood that people from a variety of countries would complete it and make comments. ${ }^{2}$ The links to the six language versions of the survey were sent to professional organizations, language service providers, institutions, personal contacts, blogs and other multipliers throughout the world with a short introductory email message and the request to forward the message and links to any professional translators in their networks.

A total of 1,850 respondents from almost 50 countries completed the survey within the 4.5 months that it was available online (from mid-August to December 2014). All of them first had to read and agree with the consent form presented at the beginning of the survey and be at least 18 years of age. There were only a limited number of compulsory items, and respondents could skip any of the other questions. This was intended to maximize the chances that respondents would continue to the end of the survey, but the consequence was that some questions were answered by fewer respondents than others (for example, a question about workflow software, which immediately preceded the questions about CAT tools, was answered by 1,782 of the 1,850 survey respondents). In addition, some specific items and comment fields were only made available if the response to a previous item indicated that it was relevant. The number of possible respondents to the survey items about CAT tools and the answer choices are provided in Table 1, organised by reported mode of employment (i.e. freelancer, institutional or commercial staff translator). The items related to irritating and missing features (indicated in bold in Table 1) elicited comments from a surprisingly large number of respondents and are the focus of the analyses reported in the present paper. ${ }^{3}$

2 For convenience, the English wordings of the survey items are used here. The other language versions can be found at https://gibbon.zhaw.ch/limesurvey/index.php/257673/lang-en. For more details about other aspects of the survey and results, see Ehrensberger-Dow et al. (2016).

3 All but five of the respondents could be attributed to one of these three categories on the basis of their answer choice or self-described employment status in the comment field beside the category 'other'. 


\begin{tabular}{|l|c|c|c|c|}
\hline Survey items and answer choices & Freelancers & Institutional & Commercial & TOTAL \\
\hline $\begin{array}{l}\text { I work with the following number of CAT tools: } \\
\text { none, } 1,2,3,4+\end{array}$ & 1,430 & 244 & 163 & 1,843 \\
\hline $\begin{array}{l}\text { Using CAT tools helps me in my work: } \\
\text { never, rarely, sometimes, mostly, always }\end{array}$ & 1,011 & 201 & 130 & 1,344 \\
\hline $\begin{array}{l}\text { I switch between CAT tools: } \\
\text { never, rarely, sometimes, mostly, always }\end{array}$ & 1,011 & 201 & 130 & 1,344 \\
\hline $\begin{array}{l}\text { Switching between CAT tools disrupts my } \\
\text { productivity: } \text { never, rarely, sometimes, mostly, always }\end{array}$ & 374 & 43 & 44 & 462 \\
\hline $\begin{array}{l}\text { I prefer to: use the default setting; customise my CAT } \\
\text { tools(s) }\end{array}$ & 961 & 186 & 120 & 1,268 \\
\hline $\begin{array}{l}\text { I customise the following aspects of my CAT tool(s): } \\
\text { layout; colours; font type; tag visibility }\end{array}$ & 443 & 74 & 67 & 584 \\
\hline $\begin{array}{l}\text { Other (optional comment field about other } \\
\text { customising) }\end{array}$ & 88 & 9 & 14 & 111 \\
\hline $\begin{array}{l}\text { There are things that irritate me about the CAT tools I } \\
\text { use: yes, no }\end{array}$ & 823 & 162 & 110 & 1096 \\
\hline $\begin{array}{l}\text { Please explain what. (optional comment field about } \\
\text { Irritating Features) }\end{array}$ & 424 & $\mathbf{1 0 0}$ & $\mathbf{6 7}$ & $\mathbf{5 9 2}$ \\
\hline $\begin{array}{l}\text { There are CAT tool features I would like to have } \\
\text { which are not currently available in the tools I use: } \\
\text { yes, no }\end{array}$ & 657 & 104 & 78 & 840 \\
\hline $\begin{array}{l}\text { Which ones? (optional comment field about Missing } \\
\text { Features) }\end{array}$ & & & & $\mathbf{2 7 2}$ \\
\hline
\end{tabular}

Table 1. Questions related to CAT tools, answer choices, and number of respondents

If the response to the item "There are things that irritate me about the CAT tools I use." was "yes", then a text box opened and the respondents were asked to explain their answer with no restrictions on the length of their comments. The statement that directly followed this text box was related to features the respondents would have liked to be available in their CAT tools (i.e. "There are CAT tool features I would like to have which are not currently available in the tools I use."). Again, the participants could answer by ticking the Yes or No option and were asked to elaborate on their answer if they agreed with the statement. These comments, usually provided in the same language as the survey (i.e. English, German, Spanish, French, Italian, and Portuguese), were translated if necessary and analysed using topic coding, as explained in the next section.

\subsection{Defining codes and categories}

Morse/Richards (2002: 115) define three different types of coding: descriptive coding, attributing information to bits of data, topic coding, creating categories and linking codes, and analytic coding, which takes coding a step further from topic coding by questioning the data according to how new ideas can be created in new codes (Morse/Richards 2002: 119). The methodology applied in this paper is mainly based on topic coding. This bottom-up approach means that the codes emerge from the data and are not imposed from external knowledge, e.g. previous research or presuppositions. This type of coding also involves analytical aspects in the sense that those codes are not just linked to the data but also that the data is continuously reassessed and thereby new codes are generated.

The first step in developing the codes was to label every comment with a characterisation related to it, with multiple coding of the same comment possible. This step was carried out manual- 
ly. The resulting coded comments either related to 'Irritating Features' ( $\mathrm{n}=931$ ) or to 'Missing Features' $(n=329)$ and so were grouped into one of these two sets. ${ }^{4}$ After labelling the comments, the next step was to find clusters of similar codes to eventually form categories. This involved reviewing, changing and altering the labels as well as the codes. Once a code was defined, it was tested against the data by checking how often it was mentioned.

A major step for establishing final codes was the development of a codebook, which contained all the codes and descriptions of how the codes should be understood and applied. The codes were worked out in collaboration with all authors of the paper. The codes that emerged for Irritating Features were grouped into the following categories: Technical Performance; CAT Tool Functionalities; Text Appearance; User Interface; Organisational Aspects; Compatibility (see the code labels and descriptions in Tables 2-7).

\begin{tabular}{|l|l|}
\hline Code & Description of Technical Performance codes \\
\hline Crashing & Tool breaking down, crashing or freezing \\
\hline Lagging & Tool functioning slowly and not reacting to commands as quickly as needed \\
\hline Bugs/errors & $\begin{array}{l}\text { Occurrence of errors or bugs, error messages that are unclear/difficult to } \\
\text { understand }\end{array}$ \\
\hline Access & Difficult accessing online servers or cloud based services \\
\hline Other technical performance & Any other issues with technical performance \\
\hline
\end{tabular}

Table 2. Codes for the Technical Performance category

\begin{tabular}{|l|l|}
\hline Code & Description of CAT Tool Functionalities codes \\
\hline Segmentation & $\begin{array}{l}\text { Issues with segmentation feature such as problems merging segments, } \\
\text { autopropagation and irritation caused by segmented view of text }\end{array}$ \\
\hline Concordance & Issues with concordance search feature \\
\hline Configuration/settings & Issues configuring projects or with settings in general \\
\hline TMs/Termbases & Issues with translation memories, Termbases or alignment \\
\hline Missing Features & Mentioning of features that are not available in the tool \\
\hline Other CAT Tool Functionalities & Any other issues related to CAT tool-specific functionalities \\
\hline
\end{tabular}

Table 3. Codes for CAT Tool Functionalities category

\begin{tabular}{|l|l|}
\hline Code & Description of Text Appearance codes \\
\hline File-type related & Corrupted formatting due to file type related issues, preview/overview feature \\
\hline Formatting & Issues with font, text size, tags, capitalisation, or numbers \\
\hline Other text appearance & Any other issues regarding formatting and text appearance \\
\hline
\end{tabular}

Table 4. Codes for Text Appearance category

4 Note that one respondent may have made several comments in one, which is why these numbers are greater than the total number of responses in Table 1. 


\begin{tabular}{|l|l|}
\hline Code & Description of User Interface codes \\
\hline Shortcuts & $\begin{array}{l}\text { Issues with shortcuts, for instance if the tool does not offer the possibility of } \\
\text { creating them }\end{array}$ \\
\hline Complexity & Complaints that the tools are too complicated, complex or not intuitive \\
\hline Customisation & Issues with level of customisation available \\
\hline Physical ergonomics & $\begin{array}{l}\text { Physical ergonomic issues such as eye strain; issues such as the tool requiring the } \\
\text { use of hardware such as a mouse }\end{array}$ \\
\hline Layout & $\begin{array}{l}\text { Mentions of fussy, busy displays or screens, too many windows, buttons, menus } \\
\text { etc. }\end{array}$ \\
\hline Other UI issues & $\begin{array}{l}\text { Issues with the layout of the text on the screen (e.g. horizontal or vertical display } \\
\text { of ST and TT); any other issues with user interface }\end{array}$ \\
\hline
\end{tabular}

Table 5. Codes for User Interface category

\begin{tabular}{|l|l|}
\hline Code & Description of Organisational Aspects codes \\
\hline User support & $\begin{array}{l}\text { Not being able to find solutions to issues in manuals, help guides, handbooks, } \\
\text { online support, forums etc. }\end{array}$ \\
\hline Cost & Expense of tools, licences, upgrades \\
\hline Training/knowledge acquisition & Need for further training, e-learning etc. \\
\hline Imposed usage & $\begin{array}{l}\text { Irritations as certain CAT tools are imposed on translators by clients or it is } \\
\text { necessary to use tools created by clients }\end{array}$ \\
\hline Other organisational aspects & Any other organisational aspects \\
\hline
\end{tabular}

Table 6. Codes for Organisational Aspects category

\begin{tabular}{|l|l|}
\hline Code & Description of Compatibility codes \\
\hline Language compatibility & Tool not working as desired with certain languages \\
\hline Interoperability & $\begin{array}{l}\text { Issues trying to use two CAT tools or other programs (speech recognition) at the } \\
\text { same time }\end{array}$ \\
\hline Variation between CAT tools & Issues due to differences in CAT tools \\
\hline Variation between versions & Issues due to differences between older and newer versions of the same CAT tool \\
\hline File compatibility & Issues with importing/exporting certain file types \\
\hline Other compatibility & Any other compatibility issues \\
\hline
\end{tabular}

Table 7. Codes for Compatibility category

Finally, the category "Other" was used to classify any comments that were not specifically related to CAT tools or were too general to be covered by the codes outlined above. These comments are not included in the analyses of the results. All of the comments were coded using HyperResearch qualitative analysis software while further analysis of the data was done in Microsoft Excel.

The process for coding the comments about Missing Features was similar to that of coding for Irritating Features, though it was deemed unnecessary to group the codes into categories for Missing Features due to the lower number of comments. Table 8 presents the codes for Missing Features and a description of what each code means. 


\begin{tabular}{|l|l|}
\hline Code & Description \\
\hline Improvements & Improvements on already existing features \\
\hline Compatibility & Making CAT tools more compatible with other software \\
\hline Formatting & More or new formatting features \\
\hline Workflow & Features that would alter the translation workflow or change organisation aspects of it \\
\hline Quality Assurance & QA features such as grammar or spell checker \\
\hline Search function & New search features within tool or online \\
\hline $\begin{array}{l}\text { Segmentation/ } \\
\text { alignment }\end{array}$ & Lack of alignment options or possibilities to alter segmentation \\
\hline TM/terminology & New TM features or ways of handling terminology \\
\hline Automation & Transfer of tasks to the CAT tool \\
\hline Preview & Lack of preview feature \\
\hline Customisation & Ability to personalise buttons or customise the tool according to one's needs \\
\hline
\end{tabular}

Table 8. Codes for Missing Features

In order to ensure consistency, the initial coding was done separately by two of the authors with the data sets in three of the languages. The comments in the other three languages were coded by one of the authors and spot checked by native speakers of the respective language who had been instructed in the coding methodology. The results of the spot checking were then compared with the initial coding and any discrepancies were discussed and resolved. A total of $10 \%$ of the codes were checked this way, with a consistency rate of over $90 \%$. Apart from the findings of this survey (presented below), the robust coding and resulting categorisation was found to work well for data analysis and we are optimistic that this could be re-used by other researchers who wish to investigate CAT technology in a similar way.

\section{Results}

A brief general overview of the quantitative results of the items related to CAT tools in the ErgoTrans survey is given below before the detailed analyses of the Irritating Features and Missing Features are presented.

\subsection{Overview of survey items related to CAT tools}

The first question related to CAT tools concerned the number that the respondents used (see Figure 1), with proportionately fewer freelancers than commercial and institutional translators using the tools (i.e. $71 \%$ vs. $80 \%$ and $82 \%$, respectively). ${ }^{5}$ As reported elsewhere, practically all of the CAT users find them helpful at least sometimes (for more details, see Ehrensberger-Dow et al. 2016). For those who use more than one tool and at least sometimes switch between CAT tools, more than half $(59 \%)$ said that it was disruptive to their productivity at least some of the time. Perhaps to avoid such disruptions to their cognitive flow, almost half of the respondents to the next survey item (44\%) said that they prefer to customise aspects of their CAT tools instead of simply using the default settings.

5 Consistent with this lower uptake of translation technology, far fewer freelancers used workflow software relative to the other two groups ( $24 \%$ vs. $53 \%$ of the commercial and $57 \%$ of the institutional translators). 




Figure 1. Number of CAT tools used by respondents

A closer examination of the aspects of CAT tools typically changed by respondents who do not use the default settings indicates that layout is by far the most problematic feature, although tag visibility and the default font type also seem to be non-optimal (Table 9). Of the aspects listed in the optional comment field that opened if "Other" was chosen, font size and keyboard shortcuts were mentioned most often. The responses to this survey item provide some information about the relative importance of certain aspects of CAT tools for the translators who are familiar enough with their tools to customise them, but the comments about irritating features analysed in the next section are much more revealing with respect to potential for cognitive friction.

\begin{tabular}{|l|c|c|c|c|}
\hline Aspects customised & Freelancers & Institutional & Commercial & TOTAL \\
\hline Layout & $81 \%$ & $88 \%$ & $84 \%$ & $82 \%$ \\
\hline Colours & $45 \%$ & $38 \%$ & $51 \%$ & $45 \%$ \\
\hline Font type & $63 \%$ & $49 \%$ & $52 \%$ & $60 \%$ \\
\hline Tag visibility & $63 \%$ & $74 \%$ & $76 \%$ & $66 \%$ \\
\hline Other & $20 \%$ & $12 \%$ & $18 \%$ & $19 \%$ \\
\hline
\end{tabular}

Table 9. Aspects of CAT tools customised by respondents

\subsection{Irritating Features}

Of the 1,096 CAT tools users who responded to the item about irritating features, 59\% said that there were features that irritated them, and $92 \%$ of those respondents took the time to provide comments to explain exactly what they were. The length and explicitness of the comments ranged from only a couple of words covered by one code (e.g. "Often too complicated.") to those that mentioned several different aspects covered by more than one code (e.g. "Not user-friendly. Too many clicks. Unintelligible error messages"). The longest comment was ninety-four words long. This high response rate and level of detail by some respondents highlights how important cognitive friction caused by language technology might be in the professional translation workplace.

The percentage of coded comments allocated to each of the six categories of Irritating Features is shown in Figure 2. More than half (i.e. 53\% in total) of the issues mentioned in the comments are related either to the User Interface $(n=256)$ or to CAT Tool Functionalities $(n=236)$. The most common codes to emerge from within those categories were Complexity, Segmentation, Formatting, Layout, Bugs/Errors, Customisation, and Training, as elaborated below. 


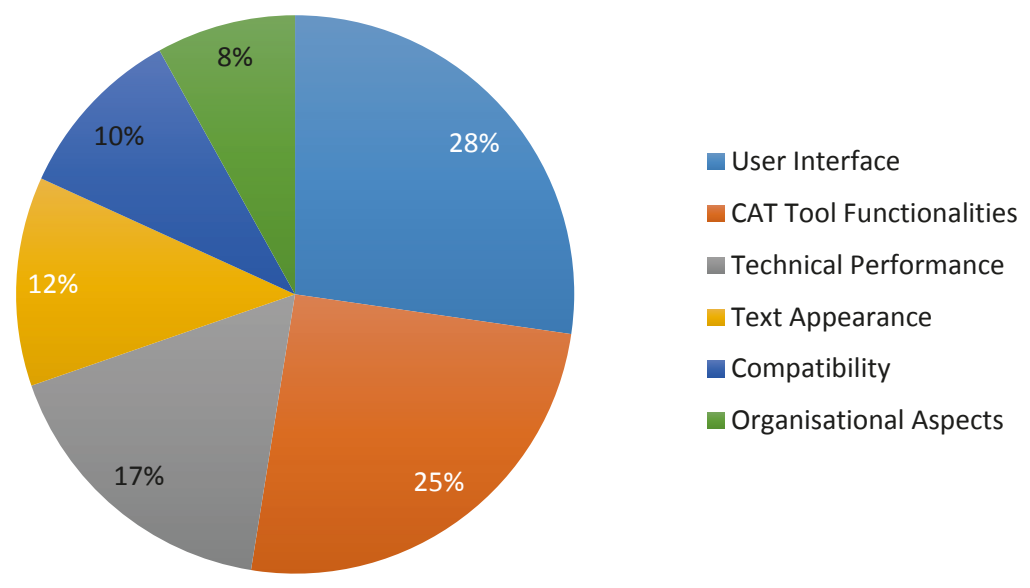

Figure 2. Percentage of comments on Irritating Features $(\mathrm{n}=931)$

\subsubsection{Most common Irritating Features}

The most commonly mentioned irritating issue was Complexity (in the User Interface category). From a total of 931 comments, this code represents 102 comments, which is $11 \%$ of the total. As described in Section 3.2, the Complexity code refers to "complaints that the tools are too complicated, complex or not intuitive". To identify what translators find complex or confusing about the CAT tools they are working with every day, those 102 comments were further divided into subcodes for the purposes of analysis. These sub-codes were not part of the original codebook but this additional coding was necessary for the further analysis of the 102 comments. The comments on Complexity either refer directly to the complexity of the tool, the tool not being intuitive for navigation or not user friendly, and the number of clicks needed to complete certain tasks.

The second most irritating feature identified by translators was Segmentation of the source and target text. Of the 931 comments, 83 referred to this issue. Within these 83 comments, the difficulties that were referred to included not only the fact that the text is displayed in a segmented way but also related issues such as the lack of options for merging and splitting segments, having an obstructed view of the text, which in turn disrupts the workflow, and problems with the autopropagation feature.

The third most irritating feature was Formatting. This included problems with "font, text size, tags, capitalisation, or numbers". In total, 75 comments referred to such issues. The main issue was that certain aspects regarding the format could not be changed, e.g. the font size could not be altered or the tool did not provide the option to highlight any parts of the source or target text. Furthermore, the tags used in most of the CAT tools seem to be an issue for many translators. A number of comments mentioned difficulties with deleting or copying tags or wrong tags that corrupted the target text, making it difficult to create or export a final version of the translation. Other issues related to this code were quotation marks, especially when working with source and target languages that use different types of characters for these (e.g. French and English).

The Layout code, which referred to the text as well as the CAT tool user interface, identified another frequently mentioned problem. 61 comments mentioned issues with how the source and target text were visually presented on the screen or criticised the visual interface of the CAT tools in general. This included irritation due to too many windows on the screen, too much distraction caused by an overcrowded layout or menu as well as issues with the horizontal or vertical display of the source and target text. Another aspect of the CAT tools that was often mentioned in 
the comments was features related to translation memory and terminology. This code referred to "issues with translation memories, terminology bases or alignment". These were mentioned 56 times and included comments on the suggested results from the translation memory, fuzzy matches, and the integration (or lack thereof) of the terminology tool.

Fifty-six comments were assigned to the code Bugs/Errors. Comments with this particular code referred to issues with technical bugs, i.e. malfunctions of the software, as well as incomprehensible error messages.

Although it had already been addressed in a specific item earlier in the survey, Customisation was a code that was used 30 times. The comments allocated to this code pointed out the lack of freedom and options in customising the CAT tool to the needs of the translators.

Another aspect worth mentioning is the code Training. This code refers to comments mentioning the lack of knowhow or the need for more training with CAT tools. Even though comments like this were not made as often as those captured by the codes described at the beginning of this section, they are still important as they can function as links between different issues and provide additional information on what irritates translators about their tools.

Three other categories, Text Appearance (115), Compatibility (94) and Organisational Aspects (72), also represent an important part of the issues translators encounter when working with CAT tools, but cannot be elaborated in greater detail due to the limitations of this paper. Therefore, further analysis and the following discussion of the results will be largely based on the previously mentioned categories and their corresponding codes.

\subsubsection{Comparing modes of employment}

Freelance, institutional and commercial translators all work in different environments with different challenges and benefits in terms of CAT tools. While commercial and institutional translators can usually rely on a team of IT specialists and co-workers who are constantly around to provide support, freelancers mainly work alone and have to rely on their own expertise. Furthermore, the whole translation process differs between these types of translators in terms of scope, agents involved as well as available resources. It is therefore interesting to compare whether these different types of working environments and challenges have an influence on the issues translators encounter in CAT tools. This was the motivation for the second research question: What influence does the working environment have on the perception of these negative aspects?

When asked if there were aspects of their CAT tools that irritated them, the groups differed in their responses: just over half (56\%) of freelancers said yes, whereas even more of the institutional and commercial translators said that there were (69\% and $65 \%$, respectively). Disproportionately more of the comments on irritating features were also made by the institutional and commercial translators $(17 \%$ and $11 \%$ compared to their representation in the survey as a whole of $13 \%$ and $9 \%$, respectively).

The main issue mentioned by all three employment groups (freelance, institutional and commercial translators) was, as highlighted above, the Complexity of the tool. In terms of the different codes, there are only minor differences among them. Besides Complexity, in all three groups, Segmentation, Translation Memory and Layout are ranked as the most common issues. Interestingly, commercial translators did not comment as often on formatting issues as the freelance and institutional translators did. While Formatting is the second most frequently mentioned issue for institutional translators and the third most frequent for freelancers, it was only mentioned in $5 \%$ of the comments from commercial translators.

An interesting and, to a certain extent, surprising result is the number of comments from commercial translators concerning the Technical Performance of their computers and workstations. A total of $25 \%$ of the comments from commercial translators were related to technical aspects such as bugs, error messages or other technical difficulties. In comparison, freelancers (14\%) and institutional translators $(15 \%)$ displayed a much lower comment rate on this particular issue. This 
is surprising, as commercial translators usually work in an environment that provides them with a well-trained team of information technologists and a good technical infrastructure. Freelance translators, on the other hand, have to build and maintain their own infrastructure, which is often just a single computer or laptop, and they need to know how to solve technical problems on their own. The reasons for this discrepancy might lie in the complexity of the working environment and the translation process of translation agencies and companies.

The issues with the User Interface are another aspect where the results show substantial differences between the modes of employment. Having established this area as one of the main issues mentioned by all the participants, it is not surprising that the percentages of comments on this category by freelancers and commercial translators are as high as $22 \%$ and $18 \%$ respectively. However, the high percentage of comments on this by the institutional translators $(32 \%)$ suggests that the issues related to the user interface need to be addressed in particular for this cohort. The difference is primarily due to the much larger relative proportion of Complexity and Layout codes for the institutional translator compared with the results from freelancers and commercial translators.

For the category of CAT Tool Functionalities, the greatest difference can be seen when comparing commercial (28\%) and institutional translators (17\%), while the rate of comments from freelancers lies between these two groups (24\%). The most frequent codes related to this category are Segmentation and Translation Memory, mentioned by all three groups. The categories Compatibility and Organizational Aspects account for 4 to $11 \%$ of the comments over all the modes of employment. Worth mentioning here is the category Text Appearance. Overall, this category only accounted for $12 \%$ of the comments. However, for the institutional translators this seems to be a greater issue (17\% of the comments) than for freelancers $(10 \%)$ and commercial translators $(11 \%)$.

\subsubsection{Comparisons across age}

Respondents were asked to identify which age group they belonged to from the ranges displayed in Table 10. We were interested in finding out if there were any differences in opinions about CAT tools across age groups. However, the distribution of comments on Irritating Features across age groups is very similar to that of the ErgoTrans survey as a whole (see Table 10). Comparing comments across the age groups did not reveal noticeable differences between them and showed a more or less coherent distribution of comments regarding all six categories of Irritating Features.

\begin{tabular}{|c|c|c|}
\hline Age group & $\begin{array}{c}\text { Overall ErgoTrans } \\
\text { survey }\end{array}$ & $\begin{array}{c}\text { Comments on Irritating } \\
\text { Features }\end{array}$ \\
\hline $18-25$ & $3 \%$ & $3 \%$ \\
\hline $26-35$ & $26 \%$ & $25 \%$ \\
\hline $36-45$ & $26 \%$ & $29 \%$ \\
\hline $46-55$ & $27 \%$ & $27 \%$ \\
\hline $56-65$ & $14 \%$ & $14 \%$ \\
\hline $65+$ & $4 \%$ & $2 \%$ \\
\hline
\end{tabular}

Table 10. Comments on Irritating Features related to age group

There are, however, two anomalies regarding the age groups that are worth noting. The results show that the translators between the ages of 26 and 35 commented more often on Technical Performance than the other age groups did: $24 \%$ of the comments from 26-35-year-olds mentioned technical problems and issues. The percentages from the other groups vary from $11 \%$ to $16 \%$. On the one hand, this could indicate that the younger translators lack an understanding of how to handle the CAT tools when they experience technical difficulties due to their inexperience with these 
tools. On the other hand, and this seems more plausible, the younger translators who have grown up with relatively sophisticated and reliable computer technology are not as tolerant of technical flaws as the older generations who have experience of technology shortcomings from the early days of the personal computer.

Another interesting aspect is that the number of comments concerned with CAT Tool Functionalities increases with every age group. The group from 26-35 had a percentage value for comments of $21 \%$, the $36-45$ year-olds a percentage value of $24 \%$, the age group $46-55$ a value of $26 \%$ and the 56-65 year old translators a value of 33\%. This suggests that the older respondents were more irritated by CAT Tool Functionalities than younger ones, which could also indicate a lower ability to deal with such issues compared with younger respondents, many of whom presumably have undergone training in CAT tools if they took university courses in translation.

\subsection{Missing Features}

The comments on features that translators miss in their CAT tools are not as numerous as the comments on the irritating features. Of the $840 \mathrm{CAT}$ tools users who responded to the item about missing features, $272(39 \%)$ said that there were some. Of those respondents, $82 \%$ provided details, which resulted in a total of 329 coded comments allocated to the eleven codes that were outlined and explained in Section 4.2 (see Table 8). The data that these comments represent relate to the third research question: What additional features do professional translators wish to have implemented in their CAT tools?

The distribution of comments about Missing Features is similar to the distribution of those on Irritating Features. The majority (75\%) were made by freelance translators, $16 \%$ by institutional translators and $9 \%$ by commercial translators.

The specific item in the survey to which respondents were asked to respond was: There are CAT tool features I would like to have which are not currently available in the tools I use. The goal was to elicit comments about features that were not already present. However, many of the comments also covered improvements to features that already exist $(25 \%$, or 81 out of 329 comments) and some comments were coded as "other" since they did not pertain to new features or improvements. As a result, the total number of comments was lower (i.e. 219). Figure 3 shows the percentage of comments for each of the Missing Features codes.

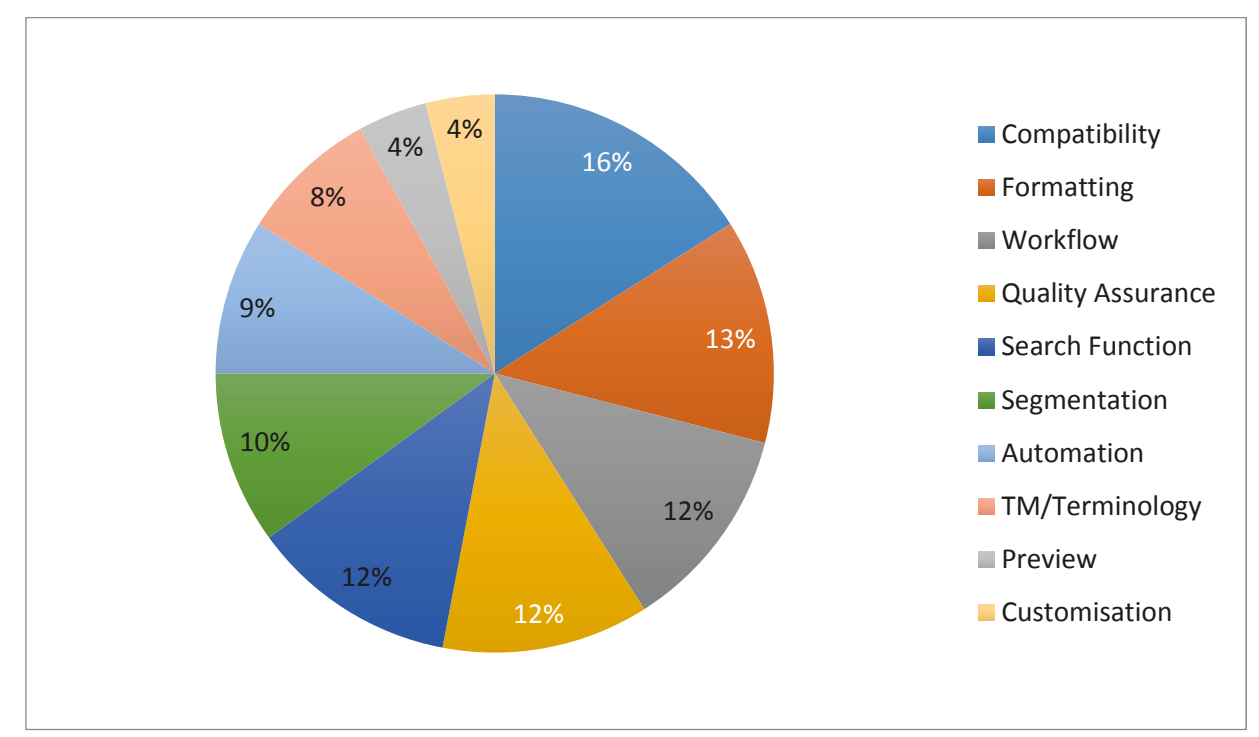

Figure 3. Percentage of comments on Missing Features ( $\mathrm{n}=219)$ 
Compatibility was the most frequently occurring code. In total, 35 comments were related to interoperability or compatibility. The topic interoperability encompasses features that allow the user to combine the CAT tool with other software such as voice recognition software or terminology management software or to work with any kind of document such as PDF, PowerPoint or Excel files. Some comments also mention the possibility of integrating with Machine Translation.

In total, 28 comments were allocated to the code Formatting. The main features mentioned in the comments included functions to highlight parts of the source or target text, options for tag handling, a feature that automatically recognises and alters quotes according to the target language, as well as the possibility of changing the font size of the source or target text. One of the participants requested a feature that would allow the creation of footnotes in the target text.

In total, 26 comments were allocated to the code Workflow. The comments proposed new features to support the organisational aspects of the translation process as well as changes to the basic structure of the CAT tools such as an interface similar to that of a more traditional text editor or working with three different languages simultaneously. The features suggested regarding organisational support included an indicator showing the percentage of already translated text, a feature for directly calculating bids/price quotations in the tool, and a deadline reminder directly integrated in the tool.

The code Quality Assurance was used 26 times and included comments related to the integration of a spell checker, a grammar checker as well as a punctuation checker. These features are already implemented in some of the tools, but as these results show, they are not a standard feature in every CAT tool.

A number of translators requested more and new Search Functions. The 26 comments related to this code included direct access to external online dictionaries and other online resources, a universal search function for all the TMs and texts that are connected to the CAT tool, as well as a find and replace function for several texts combined.

In total, 21 comments were related to the code Segmentation. The comments mainly mentioned features that are standard in many CAT tools, e.g. splitting and merging segments or the option of an alignment feature. These responses clearly indicate that altering the segments is a step many of the translators include in their work practice and that the generated segments are not always ideal.

The code TM/Terminology encompasses 18 comments but includes one particular feature that would be of great help for the translator. The feature proposed is an intelligent translation memory system that not only suggests translated segments based on match percentages but would learn from the corrections the translator makes when using the TM matches and automatically corrects the corresponding segment. Other translators wished to disable the language variants. The problem mentioned here is the fact that a TM can only be used for translations from and into the language variants defined in the TM, e.g. a TM with the variants German to British English cannot be used for a translation from German to American English.

The comments pertaining to Automation all mentioned transferring more tasks to the CAT software in order to facilitate the task at hand and reduce the translator's own mental workload. The features mentioned in the 19 comments included autosuggest, autosave, autocorrect, and automatic updates. These features certainly exist in some CAT tools but not in all of them.

The Customisation code encompasses 10 comments and is concerned with the customisation of toolbars, shortcuts or buttons the translators wish to create and change to fit to their own needs. The code was only used for 10 comments as this kind of feature does not appeal to everyone given that applying it efficiently might require a high level of technical knowledge about the tool and software in general.

In total, 9 comments were correlated with the code Preview. The participants' comments all mentioned the need for a preview feature to see the final layout of the target text while working on the translation. 
In general, the comments on missing features were mainly concerned with the usability of the tool and aimed at finding ways to make working with the tools easier and less complicated. Most of the comments are related to aspects directly involved with the translation task and only a few comments were related to adjustments to the workflow, such as going back to working in a text editor instead of a separate CAT tool.

\section{Conclusion}

The results demonstrate that professional translators still find CAT tools irritating, even after many years of deployment. More than half of the comments on irritating features were associated with the User Interface or with CAT Tool Functionalities. The most common issue mentioned was the Complexity of the UI, which, on further analysis, indicated a lack of an intuitive navigation system, lack of user friendliness and a need for too many mouse clicks to perform actions within the tool. Segmentation was also a common issue mentioned. We contend that the irritation caused by these aspects increases extraneous cognitive load, interrupts 'flow' and causes cognitive friction. These issues matter to translators and, if resolved, could reduce friction and contribute to a more positive experience for translators.

While there were no striking differences across the three groups of translators, it was surprising that $27 \%$ of the comments from commercial translators were related to the technical aspects of the tools, such as bugs or other technical difficulties. We find this surprising since commercial translators often have an IT support team in place, which is not the case for freelancers. At the same time, this could be explained by the possibility that the respondents from commercial translation companies might operate in more technically demanding environments, such as IT/localisation, which require engaging with multiple and often complicated file formats.

There were no large differences in the comments given when age of respondent was taken into account, though comments on CAT Tool Functionalities increased steadily with age. We suggest that this could indicate either a lower threshold level for irritation among the more senior translators, or a lower ability to tackle the irritating features.

Comments on missing features did not always address features that were missing, but features that exist, but could be improved on and sometimes reflected the comments made about Irritating Features. The most common codes had to do with compatibility, formatting, workflow and search support. Along with the problems of complexity of the UI and segmentation, we conclude that these features are what matter most to translators.

This survey focused on the negative aspects of CAT tools by directly asking respondents if there were irritating or missing features and to elaborate on those. We did not ask about the positive aspects of CAT tools and emphasize that there are many such aspects, such as reducing repetitive translation work and improving consistency. The research deliberately focused on the negative aspects because, as the review of related research demonstrates, there still appears to be a lack of technological maturity in the professional translation domain and we hypothesize that this causes cognitive friction in the translator's daily work. New types of CAT tools are emerging onto the market, primarily cloud-based, and often with machine translation integration. Thus far, it seems that the general model has been to develop first, and ask the user later (if at all). We propose that developers should consider working with translators to fix the irritating features and aim to not introduce new irritations as new tools emerge.

\section{Acknowledgements}

We would like to express our appreciation to the translators from around the world who participated in the survey and to acknowledge the support of the Swiss National Science Foundation in the form of an interdisciplinary grant (CR13I1_143819). We would also like to thank the anonymous reviewers for their constructive comments. 


\section{References}

Bundgaard, Kristine/Christensen, Tina Paulsen/Schjoldager, Anne 2016: Translator-Computer Interaction in Action: An Observational Process Study of Computer-Aided Translation. In The Journal of Specialised Translation 25, 106-130.

Carl, Michael/Gutermüth, Silke/Hansen-Schirra, Silvia (eds): Psycholinguistic and Cognitive Inquiries into Translation and Interpreting. Amsterdam: John Benjamins.

Christensen, Tina Paulsen/Schjoldager, Anne 2016: Computer-Aided Translation Tools: The Uptake and Use by Danish Translation Service Providers. In The Journal of Specialised Translation 25, 89-105.

Cooper, Alan 2004: The Inmates are Running the Asylum: Why Hi-Tech Products Drive Us Crazy and How to Restore the Sanity. Indianapolis: Sams Publishing.

Dam, Helle V./ Zethsen, Karen K. 2010: Translator Status: Helpers and Opponents in the Ongoing Battle of an Emerging Profession. In Target 22(2), 194-211.

Ehrensberger-Dow, Maureen 2014: Challenges of translation process research at the workplace. In MonTI Special Issue $1,355-383$.

Ehrensberger-Dow, Maureen/Hunziker Heeb, Andrea 2016: Investigating the ergonomics of the technologized translation workplace. In Muñoz Martín, Ricardo (ed.), Reembedding Translation Process Research. Amsterdam: John Benjamins, 69-88.

Ehrensberger-Dow, Maureen/Hunziker Heeb, Andrea/Massey, Gary/Meidert, Ursula/Neumann, Silke/Becker, Heidrun (2016): An International Survey of the Ergonomics of Professional Translation. In ILCEA Revue de l'Institut des Langues et des Cultures d'Europe et d'Amérique 27 [online]. http://ilcea.revues.org/4004.

Ehrensberger-Dow, Maureen/Massey, Gary 2014: Cognitive Ergonomic Issues in Professional Translation. In Schwieter, John W./Ferreira, Aline (eds.), The Development of Translation Competence: Theories and Methodologies from Psycholinguistics and Cognitive Science. Newcastle upon Tyne: Cambridge Scholars Publishing, 58-86.

Ehrensberger-Dow, Maureen/O’Brien, Sharon 2015: Ergonomics of the Translation Workplace: Potential for Cognitive Friction. In Translation Spaces 4, 98-118.

Fulford, Heather/Granell-Zafra Joaquín 2005: Translation and Technology: A Study of UK Freelance Translators. In The Journal of Specialised Translation 4, 2-17.

IEA 2016: Definitions and Domains of Ergonomics. International Ergonomics Association [online]. http://www.iea.cc/ whats/index.html (accessed 24 April 2016).

Krüger, Ralph 2016: Contextualising Computer-Assisted Translation Tools and Modelling Their Usability. In transkom 9(1), 114-148.

Lagoudaki, Elina 2006: Translation Memories Survey 2006: Users' Perceptions around TM Use. In Proceedings of ASLIB Translating and the Computer 28, London, UK. 15-16 November 2006.

Lagoudaki, Elina 2008: Expanding the Possibilities of Translation Memory Systems: From the Translator's Wishlist to the Developer's Design. Unpublished PhD dissertation. Imperial College, London, UK.

LeBlanc, Matthieu 2013: Translators on Translation Memory (TM): Results of an Ethnographic Study in Three Translation Services and Agencies. In Translation and Interpreting 5(2), 1-13.

Moorkens, Joss/O’Brien, Sharon 2016: Assessing User Interface Needs of Post-Editors of Machine Translation. In Kenny, Dorothy (Ed), IATIS Yearbook 2016, London, UK: Routledge.

Morse, Janice M./Richards, Lyn 2002: Readme First for a User's Guide to Qualitative Methods. Thousand Oaks: Sage.

Nakamura, Jeanne/Csikszentmihalyi, Mihaly 2002: Flow Theory and Research. In Snyder, C.R./Lopez, Shane (eds.), Handbook of Positive Psychology, Oxford: Oxford University Press, 89-105.

O'Brien, Sharon 2006: Eye Tracking and Translation Memory Matches. In Perspectives: Studies in Translatology 14(3), 185-205.

O'Brien, Sharon 2008: Processing Fuzzy Matches in Translation Memory Tools: An Eye-Tracking Analysis. In Jakobsen, Arnt Lykke/Mees, Inger/Göpferich, Susanne (eds), Behind the Mind [Copenhagen Studies in Language 36]. Frederiksberg: Samfundslitteratur, 79-102.

Olohan, M. 2011: Translators and translation technology: The dance of agency. In Translation Studies 4(3), 342-357.

Risku, Hanna 2002: Situatedness in Translation Studies. In Cognitive Systems Research 3, 523-533.

Robbins, Philip/Aydede, Murat 2009: The Cambridge Handbook of Situated Cognition. Cambridge: Cambridge University Press.

Schnotz, Wolgang/Kürschner, Christian 2007: A Reconsideration of Cognitive Load Theory. In Educational Psychology Review 19(4), 469-508. 
Zaretskaya, Anna 2015: User Requirements Analysis. Deliverable 2.1 of the EXPERT project, FP7 Project no. 317471 [online]. http://expert-itn.eu/?q=ESR1 (accessed 24 April 2016). 